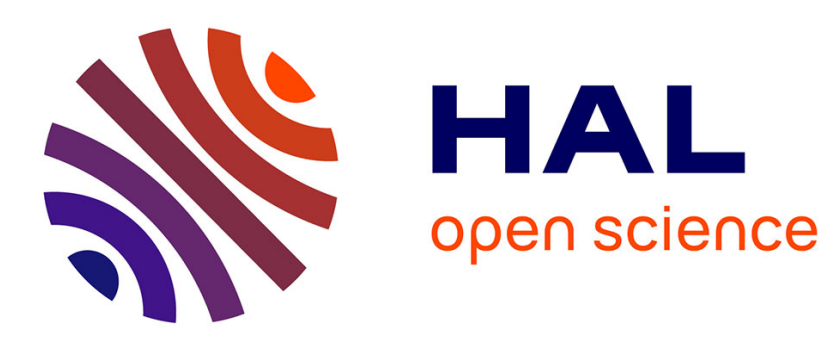

\title{
Dissymmetrization of micro-particle surface by laser-induced photochemical deposition
}

Emmanuel Hugonnot, Marie-Hélène Delville, Jean-Pierre Delville

\section{To cite this version:}

Emmanuel Hugonnot, Marie-Hélène Delville, Jean-Pierre Delville. Dissymmetrization of microparticle surface by laser-induced photochemical deposition. Applied Surface Science, 2005, 248 (1-4), pp.470-474. 10.1016/j.apsusc.2005.03.066 . hal-00094745

\section{HAL Id: hal-00094745 \\ https://hal.science/hal-00094745}

Submitted on 15 May 2009

HAL is a multi-disciplinary open access archive for the deposit and dissemination of scientific research documents, whether they are published or not. The documents may come from teaching and research institutions in France or abroad, or from public or private research centers.
L'archive ouverte pluridisciplinaire HAL, est destinée au dépôt et à la diffusion de documents scientifiques de niveau recherche, publiés ou non, émanant des établissements d'enseignement et de recherche français ou étrangers, des laboratoires publics ou privés. 


\title{
Dissymmetrization of Microparticle Surface by Laser-Induced Photochemical Deposition
}

\author{
Emmanuel Hugonnot ${ }^{\#}$, Marie-Hélène Delville ${ }^{\dagger}$, and Jean-Pierre Delville ${ }^{\#}$ \\ \#Centre de Physique Moléculaire Optique et Hertzienne, UMR CNRS/Université No.5798, \\ Université Bordeaux I, 351 Cours de la Libération, F-33405 Talence cedex, France \\ ${ }^{\dagger}$ Institut de Chimie de la Matière Condensée de Bordeaux, UPR CNRS No 9048, 87 Avenue \\ du Docteur A. Schweitzer, F-33608 Pessac cedex, France
}

Corresponding author: Jean-Pierre DELVILLE

Centre de Physique Moléculaire Optique et Hertzienne, UMR CNRS/Université No.5798, Université Bordeaux I, 351 Cours de la Libération, F-33405 Talence cedex, France Phone: (33) 5400022 07; Fax: (33) 540006970

Email: jp.delville@cpmoh.u-bordeaux1.fr

\begin{abstract}
We investigate the surface dissymmetrization of micro-sized particles by using the photochemical reduction of chromate ions induced by a strongly focused continuous $\mathrm{Ar}^{+}$laser wave to nucleate and grow deposits onto one hemisphere of silica beads dispersed in the chromate solution. Growth of dissymmetric coatings is performed at $\lambda=514 \mathrm{~nm}$ with laser fluences varying from 1.3 to $10.1 \mathrm{~kW} / \mathrm{cm}^{2}$. Rescaling of the measured deposit growth laws reduces to a single master behavior predicting the desired dissymmetry. The technique can be used to tailor micro-scale dissymmetric patterns by scanning the beam.
\end{abstract}

PACS numbers: 81.15.Fg, 81.10.Dn, 42.40.Eq, 42.70.Gi 
Keywords: photochemical deposition, surface patterning, nano/micro-sized particles, laser, chromate

\section{Introduction}

Nano- and micro-size particles have an increasingly important role in emerging nano/micro "technologies". Many efforts have been made to prepare isotropically coated and functional [1] colloid particles. Surprisingly, much less attention has been dedicated to the dissymmetrization of their chemical properties, since dissymmetry brings an additional degree of freedom that can be used to increase the complexity of targeted micro/nanomaterials. Such a strategy could be very promising for self-assembling, [2], chemical sensoring, [3], drug delivery, [4], or micro-optics [5]. However, conventional and advanced lithographic techniques [6] are essentially limited to planar and cylindrical substrates [7]. The first attempts for particles dissymmetrisation were investigations of partial silanization [8], chemisorption in directional fluxes [9] and differential nano-clusters adsorption at interfaces [10]. We extend these methods by presenting a general and convenient alternative that (i) combines micro-photochemical deposition driven by a strongly localized laser excitation, to "nucleate” a deposit, i.e. a dissymmetry, on the surface of a micro-sphere and (ii) control its growth by irradiation parameters. When properly rescaled, the coating growth laws measured for different excitations reduces into a single master curve helping to control and predict the desired dissymmetry. The versatility of the technique and its ability to tailor micro-scale patterning on only one of the two hemispheres are also described.

\section{Experimental Section}


Laser chemical processing is a powerful non-contact tool for micro-machining and micro-processing of materials [11]. Photo-chemically stimulated deposition represents nowadays an attractive alternative for direct material writing without using mask-based photolithography. The technique uses UV or visible photons to efficiently break chemical bonds into molecules and induce rapid thin film deposition via very moderate beam intensities. Furthermore, laser light offers localized processing by accurate spatial excitation and spectral selectivity in molecule activation. When performed in liquids, photochemical deposition can be applied to a broad range of precursors, including molecular compounds. Moreover, since the light energy conversion yields are much higher than in gas phase, one requires relatively low power lasers for processing [12].

Based on the photo-reduction of $\operatorname{Cr}(V I)$ ions into $\operatorname{Cr}(I I I)$, used in dichromated gelatins for optical recording [13], the experiment, shown in Figure 1, is based on the photochemical deposition of a chromium oxide layer [14]. It is performed at room temperature on $10 \mu \mathrm{m}$ NUCLEOSIL ${ }^{\circledR}$ silica beads dispersed in a liquid mixture composed of potassium chromate, ethanol, hydrochloric acid and water. The samples are enclosed in a tight very thin home-made cell, composed of two glass slides separated by $30 \mu \mathrm{m}$ thickness mylar spacers, to prevent disturbing thermal effects induced by the light absorption, such as convection or liquid boiling. The upper slide is silanized to eliminate any photochemical deposition on the entrance face of the cell. The cell is horizontally mounted to keep the beads at rest on the bottom plate. A computer controls translation stages, with a step accuracy of 0.5 $\mu m$, to move the cell holder in the $x, y$ and $z$ directions.

Two forms of $\operatorname{Cr}(V I)$ are found in aqueous solutions according to the following equation: $\mathrm{HCrO}_{4}^{-} \rightleftharpoons \mathrm{CrO}_{4}^{2-}+\mathrm{H}^{+}(\mathrm{pK}=6.49)$ [13]. At $\mathrm{pH}=3, \mathrm{HCrO}_{4}^{-}$is the major species in solution. As this hexavalent form of chromium can be activated by light in the 
blue-green wavelength range, we used a $A r^{+}$laser $(\lambda=514 \mathrm{~nm})$. The addition of an organic compound, such as an alcohol ( $\mathrm{ROH}$ ), enhances the photo-redox process of $\mathrm{HCrO}_{4}^{-}$to amorphous chromium hydroxide $\mathrm{Cr}(\mathrm{OH})_{3}$. This $\operatorname{Cr}(\mathrm{III})$ species has a very low solubility. The major pathway from $\operatorname{Cr}(V I)$ to $\operatorname{Cr}(I I I)$ starts with a light-induced excitation of $\operatorname{Cr}(V I)$ followed by reduction to a $\operatorname{Cr}(V)$ intermediate and a dark reaction from $\operatorname{Cr}(V)$ to $\operatorname{Cr}(I I I)$ [13], see Eq. 1:

$$
\begin{aligned}
& \operatorname{Cr}(V I)+h v \rightleftharpoons \operatorname{Cr}(V I)^{*}, \\
& \operatorname{Cr}(V I)^{*}+R O H \rightarrow " C r(V)^{\prime \prime} \rightarrow \ldots \rightarrow \operatorname{Cr}(I I I) .
\end{aligned}
$$

\section{Results}

According to Eqs. (1a-b), the $\operatorname{Cr}(I I I)$ concentration in solution increases with laser intensity. The nucleation of the $\operatorname{Cr}(I I I)$ precipitate occurs as soon as the solubility limit is achieved. Consequently, a $\mathrm{Cr}(\mathrm{OH})_{3}$ photo-deposit of circular shape starts to grow onto the silica particle hemisphere intercepted by the exciting beam. See the temporal sequence of spot growth for $350 \mathrm{~s}$ in Figure 1. The side view of the central bead illustrated in the experimental setup clearly shows that the deposited dissymmetry is preserved until the complete covering of one hemisphere. Considering the homogeneity of the induced deposit, we performed measurements of the photodeposit growth rate. As the substrate is curved, and growth mechanisms involve solute flux along the optical direction, the image processing approximates the measured cross section of the covered hemisphere by a circular area of radius $R(t)$ instead of considering the hemispherical surface covered by the deposit. The 
inset of Figure 2 shows measurements, carried out with a beam waist $a_{0}=0.42 \mu \mathrm{m}$ and incident laser beam power in the range $7 \leq P \leq 56 \mu W$. At a given time, the growth rate observed increases for increasing beam power.

\section{Discussion}

To interpret these data, we use a droplet growth model [15] considering the case of small normalized optical absorption $\sigma a_{0}<<1$, where $\sigma$ is the optical absorption of the chromate solution. For $\sigma a_{0}<<1$, the radial growth rate of the deposit is given by:

$$
\frac{d \rho}{d \tau}=\exp \left(-\rho^{2} / 2\right) \cdot I_{0}\left(\rho^{2} / 2\right)
$$

where $I_{0}\left(\rho^{2} / 2\right)$ is the $0^{\text {th }}$ order modified Bessel function of the variable $\rho^{2} / 2 \cdot \rho=R / a_{0}$ is a normalized radius and $\tau=\left(K^{*} P \sqrt{\pi} \cos \vartheta / 2 a_{0}^{2}\right) t$ is a reduced time, where $K^{*}$ is the normalized reaction rate for the $\operatorname{Cr}(V I)^{*} \rightarrow \operatorname{Cr}(I I I)$ reaction and $\vartheta$ is the contact angle of the deposit on the substrate. Consequently, the entire dependence in exciting beam parameters is transferred to both the rescaled deposit radius and the new thermodynamic time scale. The apparently different growth laws obtained for various conditions of optical excitations (Inset of Figure 2) point out for a single-scaled dynamic when the radius of the deposit is rescaled with $a_{0}$ and the time with $a_{0}^{2} / P$. This data reduction on a master curve is shown in Figure 2 . Note that a simple asymptotic regime emerges for $\sigma a_{0}<<1$. Indeed, using the relation $I_{0}\left(\rho^{2} / 2\right) \approx \exp \left(\rho^{2} / 2\right) / \sqrt{\pi} \rho$ for $\rho>>1$, we find $\rho \propto \sqrt{\tau}$, in good agreement with the 
observations as a power law fit of the data leads to $R(t) \propto(P t)^{0.56 \pm 0.03}$. This results shows that the growth simply depends on the energy deposited in the medium when the deposit radius becomes much larger than that the excited zone. Agreement is also observed at smaller $\rho$ for which the regime $\rho \propto \tau$ is predicted from Eq. 2. Thus, our approach clearly suggests a unified behavior of the dynamics [16].

The method can easily be extended to micro-scale patterning of particles by scanning the cell holder stages [17]. One mode consists in discontinuously realizing micro-patterns dot by dot under a finite exposure for each deposit followed by a substrate translation perpendicular to the beam axis (Figure 3a). In the second mode, the laser writes continuous lines instead of dots (Figure 3b).

Applications of asymmetric patterning of micro-spheres can be applied in many emerging areas. A direct application is to tailor the phase and the amplitude of micro-lens coatings in optoelectronics devices [18]. Another feature is the chemical anisotropy resulting from dissymmetrization of the micro-particle hemispheres. Indeed, the induced coating can be chemically modified with organic functions or used as a mask to modify the uncoated surface. In a second step, the deposit can be removed, allowing a further different functionalization of the previously covered surface [10]. Non-symmetrical micro-spheres can also be used to force self-assembling in a preferred way and build organized architectures, such as photonic crystals.

\section{Conclusion}

We used laser-assisted micro-photochemical deposition to coat and dynamically tailor the surface dissymmetry of micrometric silica beads. Considering the agreement observed between the experiments and predictions, as well as the description of the deposit growth rate 
in scaling terms, our method can easily be extended to the photochemical deposition of noble metals [19] or semi-conductors [20] on various particles such as $\mathrm{SiO}_{2}, \mathrm{Al}_{2} \mathrm{O}_{3}$ and $\mathrm{TiO}_{2}$. We believe that micro-photodeposition can be considered as an interesting novel method to be used for micro-lens dissymmetrization, microsphere functionalization and micrometric selfassembly. 


\section{Figures Caption:}

Figure 1: Schematics of the experimental setup used for investigating the radial growth of a circular coating photodeposited on one hemisphere of a micro-particle. A top view of the coating time evolution for a beam centered $10 \mu \mathrm{m}$-silica bead is shown at the bottom between $t=0$ and $350 \mathrm{~s}$. The incident beam power and the beam waist values are $P=28 \mu W$ and $a_{0}=0.42 \mu m$ respectively.

Figure 2: Plot in reduced variables $\rho=R / a_{0}$ and $\tau \propto P t / a_{0}{ }^{2}$ for the growth of the dissymmetrical photodeposited spot presented in the Inset for increasing beam power excitations. The dash-dotted line is the linear regime $\rho(t) \propto P t / a_{0}{ }^{2}$ predicted for the early growth stage while the dashed line corresponds to the power law fit $\rho(t) \propto \sqrt{P t / a_{0}^{2}}$ expected for $\rho(t)>1$.

Figure 3: Dissymmetric micro-patterns deposited on silica beads by controlled movement of the cell. a) Dot patterns realized at $P=28 \mu W$ and $a_{0}=0.42 \mu \mathrm{m}$ with a time exposure $\Delta t=5 \mathrm{~s}$; the dot size is $\sim 1 \mu \mathrm{m}$. b) Line patterns for $P=42 \mu \mathrm{W}, a_{0}=0.42 \mu \mathrm{m}$ and a scanning rate $V_{s}=1 \mu \mathrm{m} / \mathrm{s}$; line sizes smaller than $1 \mu \mathrm{m}$ can be observed. 
Figure 1

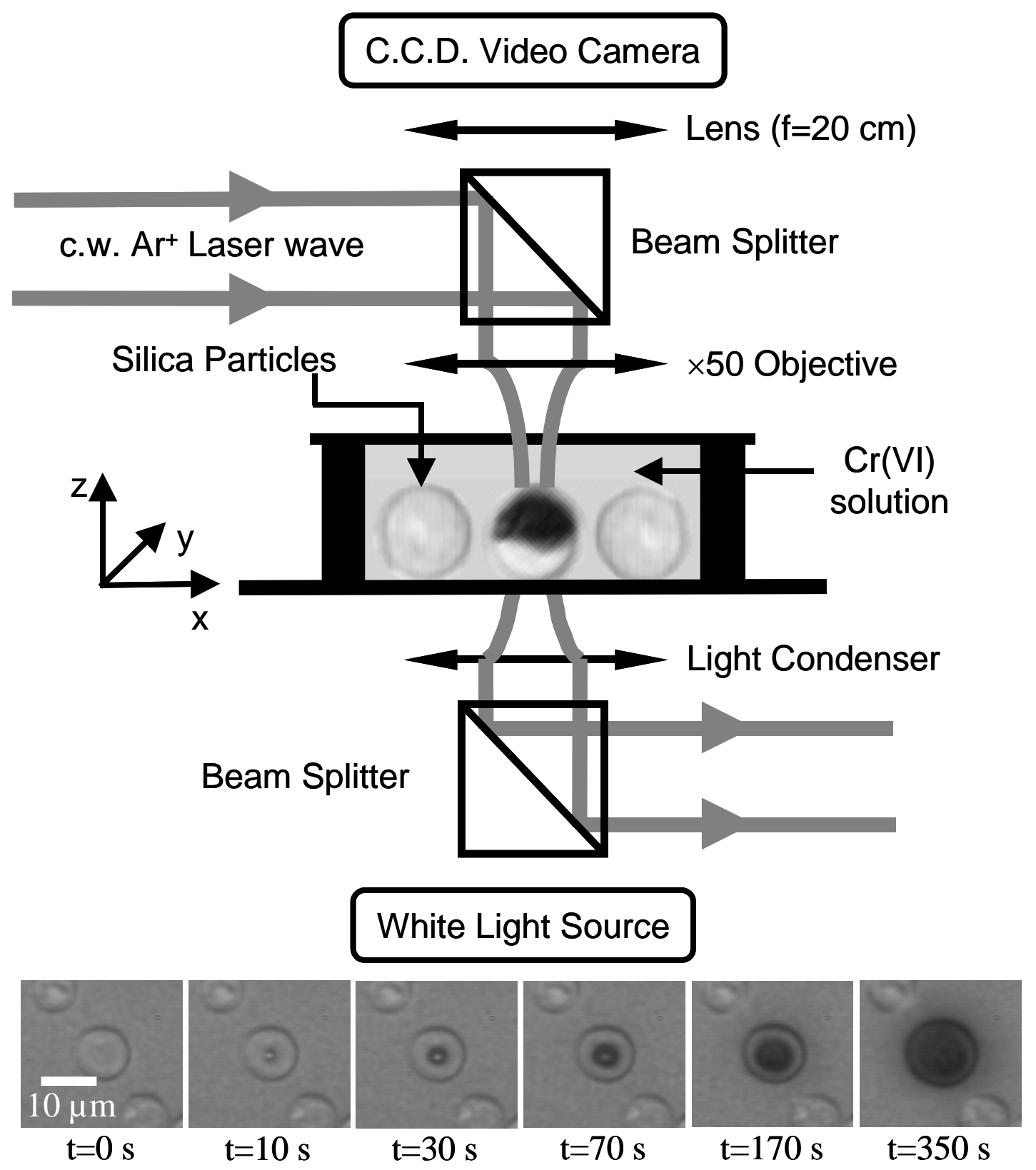


Figure 2

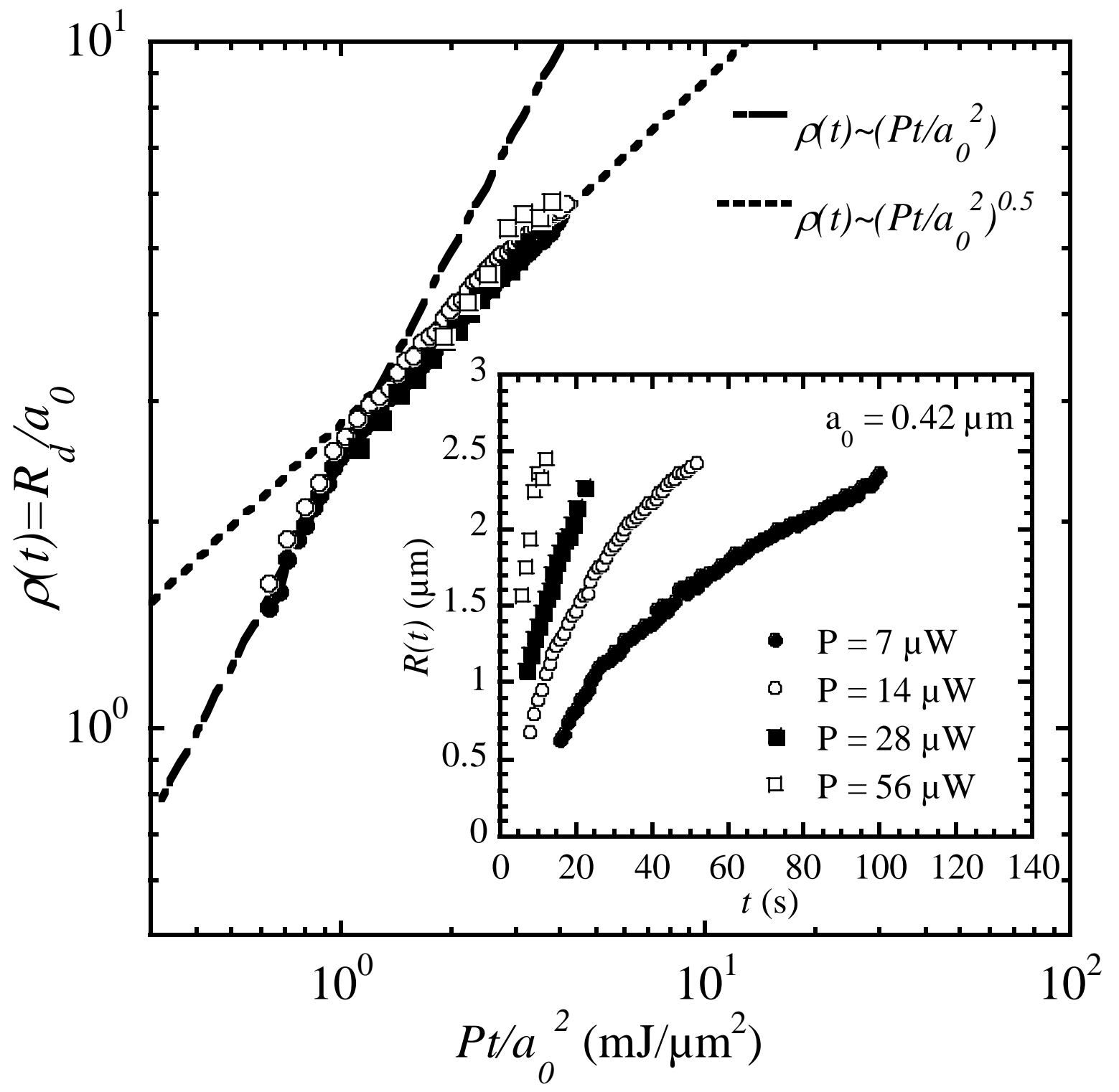


Figure 3
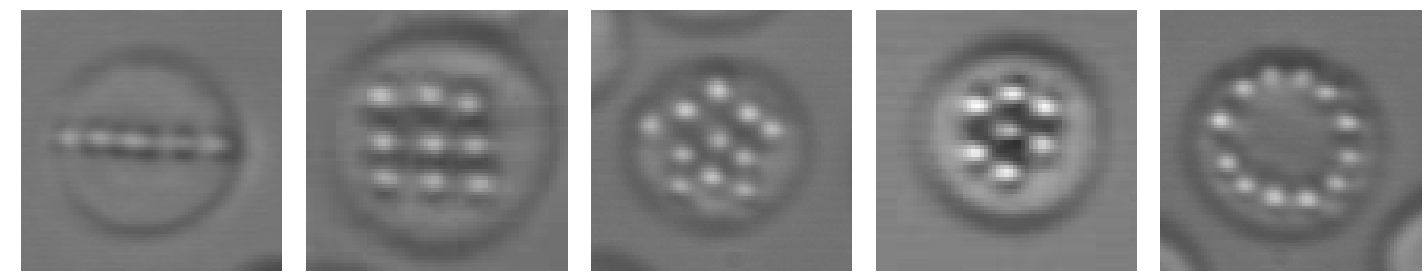

a)
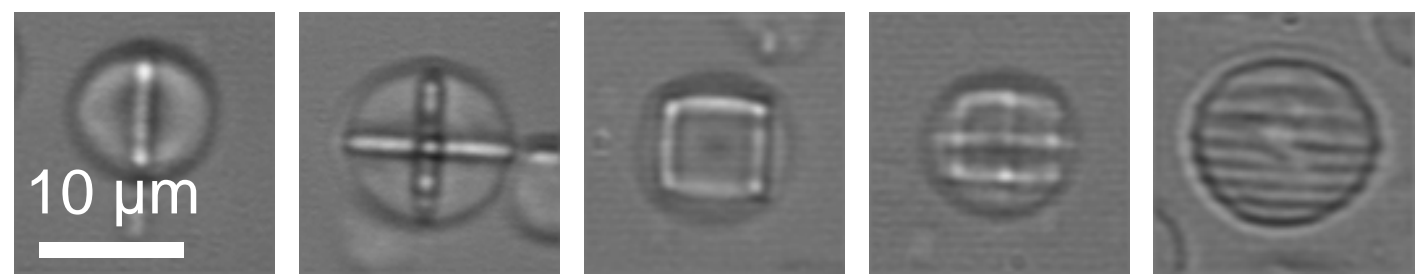

b) 


\section{References:}

[1] F. Caruso, Adv. Mater. 13 (2001) 11.

[2] J. M. Lehn, Supramolecular Chemistry: Concepts and Perspectives (VCH: Weinheim, 1995).

[3] M. Himmelhaus, and H. Takei, Sensors and Actuators B 63 (2000) 24.

[4] E. Mathiowitz, J. S. Jacob, Y. S. Jong, G. P. Carino, D. E Chickering, P. Chaturvedi, C. A. Santos, and K. Vijayaraghavan, Nature 386 (1997) 410.

[5] Y. Ito, and A. L. Bleloch, L. M. Brown, Nature 394 (1998) 49.

[6] Y. Xia, J. A. Rogers, K. E. Paul, and G. M Whitesides, Chem. Rev. 99 (1999) 1823.

[7] R.J. Jackman, J. L. Wilbur, and G. M. Whitesides, Science 269 (1995) 664.

[8] C. Casagrande, P. Fabre, E. Raphaël, and M. Veyssié, Europhys. Lett. 9 (1989) 251.

[9] H. Takei, and N. Shimizu, Langmuir 13 (1997) 1865.

[10] L. Petit, J. P. Manaud, C. Mingotaud, S. Ravaine, and E. Duguet, Mat. Lett. 51 (2001) 478.

[11] D. Bäuerle, Laser Processing and Chemistry (3rd Edition, Springer, Berlin, 2000).

[12] A. Peled, Lasers Eng. 6 (1997) 41.

[13] T. Keinonen, and R. Grzymala, Appl. Opt. 38 (1999) 7222.

[14] E. Hugonnot, A. Carles, M. H. Delville, P. Panizza, and J. P. Delville, Langmuir 19 (2003) 226.

[15] E. Hugonnot, and J.P. Delville, Phys. Rev. E 69 (2004) 051605.

[16] E. Hugonnot, A. Popescu, S. Hanifi-Kadi and J.P. Delville, Phys. Rev. E 69 (2004) 051606.

[17] A. Lachish-Zalait, D. Zbaida, E. Klein, M. Elbaum, Adv. Funct. Mater. 11 (2001) 218.

[18] M. B. Stern, Microelec. Eng. 34 (1997) 299. 
[19] M. Wehner, F. Legewie, B. Theisen, E. Beyer, Appl. Surf. Sci. 106 (1996) 406.

[20] M. Ichimura, F. Goto, Y. Ono, E. Arai, J. Cryst. Growth 198/199 (1999) 308. 